\title{
Distributed System Framework for Mobile Cloud Computing
}

\author{
K. Arul Jothy, K. Sivakumar and M.J. Delsey
}

\begin{abstract}
Today's global computing environment involves the vital role of the mobile cloud computing. Mobile Cloud Computing (MCC) is the combination of cloud computing, mobile computing and wireless networks which brings rich computational resources to mobile users, network operators, as well as cloud computing providers. So its main goal is to provide the user rich experience. Now-a-day's smart phones are facing problem like slow online speed, space insufficiency (small memory), and lower stand by time. So this problem can be overcome by placing alternating solution like placing power full battery and speed can be improved by connecting the device through blue tooth or any other wireless devices and in the cloud network the basic problem is the higher response time of nodes while performing data communication operation through co-operative cache [1]. This dominant problem can be overcome by applying the distributed system as a hub in the star topology. The distributed system is used as a central controller of the star topology. Any one of the controller among the distributed system keeps track of the status of all other devices during the communication and that device will share the status among the other entire device within its own system and it use the passive scan over the device for the communication. Another basic issue in the cloud storage is that it is not able to provide security to the data stored and accessed from it and not able to prevent from the hacker so it can be overcome by using KF sensor. [Using distributed system will efficiently maintain the failure of device without data loss].
\end{abstract}

Keywords--- Mobile Cloud Computing, Co-operative Cache, Star Topology Network, Central Controller, KF sensor.

\section{INTRODUCTION}

- Mobile cloud computing (MCC) at its simplest, refers to an infrastructure where both the data storage and data processing happen outside of the mobile device[2].

- Mobile cloud applications move the computing power and data storage away from the mobile devices and into powerful and distributed computing platforms

K. Arul Jothy, Final Year CSE, Department of Computer Science and Engineering, JCT College of Engineering and Technology, Coimbatore, India.E-mail:aruljothy22@gmail.com

K. Sivakumar, Assistant Professor, Department of Computer Science and Engineering, JCT College of Engineering and Technology, Coimbatore, India.E-mail:sivakumar.karuppan@gmail.com

M.J. Delsey, Assistant Professor, Department of Computer Science and Engineering, JCT College of Engineering and Technology, Coimbatore, India.E-mail:delsey217@gmail.com

DOI:10.9756/BIJRCE.8357 located in clouds, which are then accessed over the wireless connection based on a thin native client [2].

- Mobile devices face many resource challenges (battery life, storage, bandwidth etc.)

- Cloud computing offers advantages to users by allowing them to use infrastructure, platforms and software by cloud providers at low cost and elastically in an on-demand fashion [2].

- Mobile cloud computing provides mobile users with data storage and processing services in clouds, obviating the need to have a powerful device configuration (e.g. CPU speed, memory capacity etc), as all resource-intensive computing can be performed in the cloud [2].

\section{A. MCC Popularity}

According to a recent study by ABI Research, more than 240 million businesses will use cloud services through mobile devices by 2015 .

That traction will push the revenue of mobile cloud computing to $\$ 5.2$ billion.

Mobile cloud computing is a highly promising trend for the future of mobile computing.

Many security issues are there as different technologies including networks, databases, operating systems, virtualization, resource scheduling, transaction management, load balancing, concurrency control and memory management are used in cloud computing. Some of the security issues include data isolation, browser security, malware, flooding attack, privacy, data integrity, and protection of DATA [3].

\section{Problem for Low Battery Life of Mobile Devices}

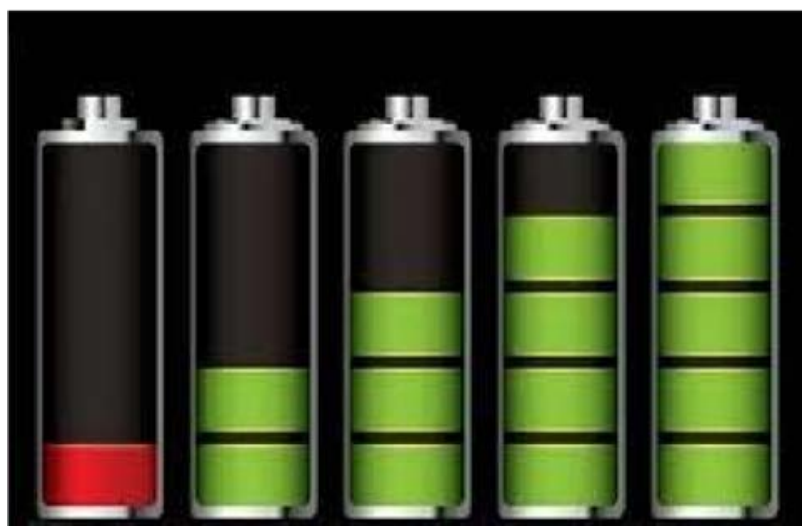

Figure 1: Low Battery Problem 


\section{2. $\quad$ Problem for Small Size of Mobile Devices}

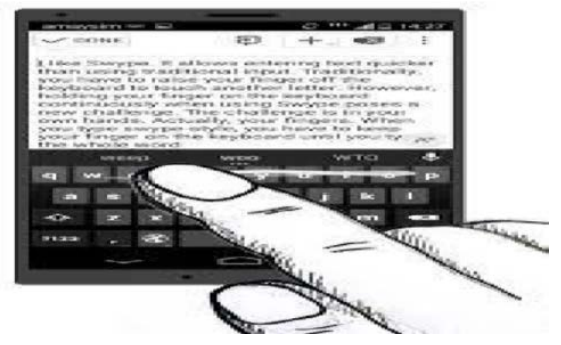

Figure 2: Small Size Problem

\section{B. Mobile Cloud Computing Architecture}

In Mobile Cloud Computing (MCC) architecture Mobile devices are connected to the mobile networks via base stations that establish and control the connections and functional interfaces between the networks and mobile devices. Mobile users' requests and information are transmitted to the central processors that are connected to servers providing mobile network services. The subscribers' requests are delivered to a cloud through the Internet. In the cloud, cloud controllers process the requests to provide mobile users with the corresponding cloud services [3].

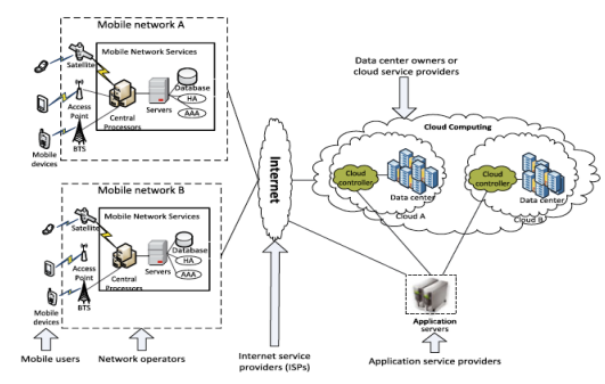

Figure 3: MCC Architecture

\section{Star Topology Network}

In a star topology, each device has a dedicated point to point link only to a central controller, usually called a hub. The devices are not directly linked to each other.

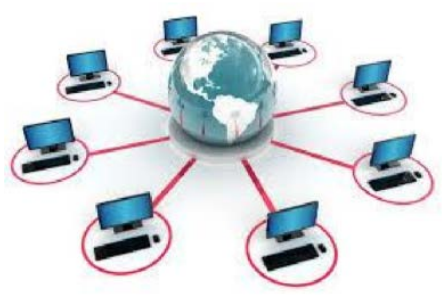

Figure 4: Star Topology Network

\section{Cloud Service Hierarchy}

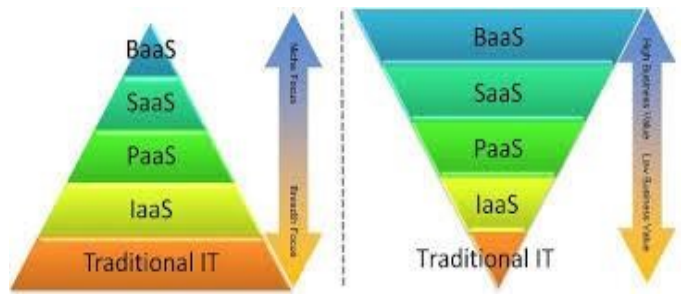

Figure 5: Cloud Service Hierarchy a) IAAS: IAAS provides storage, hardware, servers and networking components to the user. For example elastic cloud of Amazon [2].

b) PAAS: PAAS provides an environment of parallel programming design, testing etc. For example Google App engine [2].

c) SAAS: SAAS provides some applications that can be used via Internet and is paid according to the usage. For example Google Online Office [2].

\section{DePloyment Models}

a) Public Cloud: A type of cloud in which the cloud services are delivered over a network which is open for public usage [2].

b) Private Cloud: A type of cloud that is implemented on a cloud-based secure environment that is safeguarded by a firewall. Private cloud as it permits only the authorized users can use the data [2].

c) Community Cloud: A type of cloud in which the setup is mutually shared between many organizations that belong to a particular community [2].

d) Hybrid Cloud: A type of cloud which is integrated. It can be an arrangement of two or more cloud servers, i.e. private, public or community cloud that is bound together but remain individual entities [2].

\section{A. WLAN Scanning Process}

a) Active Scan: In Active Scanning a station finds out network rather than waiting for network to announce its availability to all the stations.

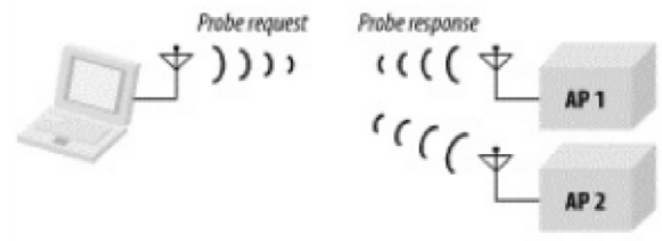

Figure 6: Active Scanning Process

b) Passive Scan: In Passive Scanning, WLAN station moves to each channel as per channel list and waits for beacon frames. All these frames are buffered and are used to decode and extract information about base stations.

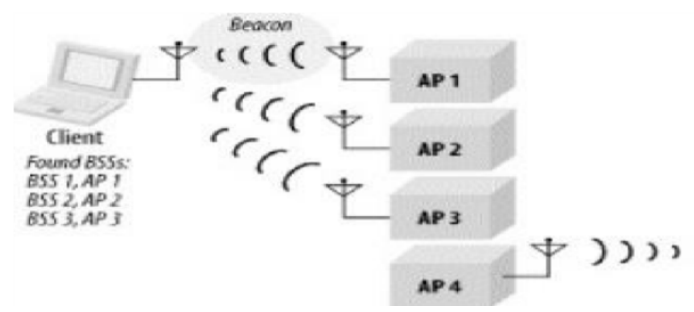

Figure 7: Passive Scanning Process

\section{B. KF Sensor}

$\mathrm{KF}$ sensor is the intrusion detection system, Which act as a honeypot to attract and detect hackers or the other unauthorized user by creating a virtual vulnerable system and services. 


\section{PROPOSED WORK}

The work proposed along with the distributed system over MCC is use to overcome the various issues faced by it.

The Limitations of today's smart phones are mainly of smaller size and low battery life. While typing on mobile devices, it often creates problem due to small size of typing pad in mobile device. To overcome the problem of the size, the mobile phone can be used via touchpad device connected through WIFI hotspot. A touchpad device can be used, where processor and storage both will be in the mobile device but virtually we can use the touchpad while typing in mobile devices. The touchpad device can be useful while travelling in vehicles. Even many more touchpad devices can be used to get the benefit of parallel computing in a single mobile device.

Second problem is low battery life of mobile devices. To overcome this problem low battery can be replaced by a powerful battery as required for the mobile device so that it can provide backup for at least 3-4 day. In this case cost will increase but the performance, battery backup, user attractiveness will also increase.

In cloud computing, instead of co-operative caching one of the devices can be chosen as a central device called 'Distributed Controller' that keeps track of the status of all the other devices connected with it using a star topology network. During this time the Central Controller [Distributed system] scan passively all the devices dynamically to get the current status about storage whereas a device need to store any data will perform an active scan over the Central Controller [Distributed system] to know the status of all other devices. As soon as, it gets the information it will proceed directly onto that particular device to store the data. Thus Response time and efficiency can both be effective and valuable for future course.
And while storing and retrieving the information from the cloud the security for the data is not provided. The hackers can easily access the data from the cloud. In order to overcome this security issue we can use the KF sensor in between user node and the cloud. So the KF sensor will categorize the authorized user and the unauthorized user (hacker) to monitor the activity. When unauthorized user access the network to collect the data it will monitor the hacker activity and it does not allow them to access the data or the resource from the cloud. The KF sensor will block the hacker to access the data from the cloud.

\section{ALGORITHM}

1. A finite set of nodes $S=\{N 1, N 2, N 3, \ldots \ldots \ldots . .$.

2. Central Controller [distributed system] (N) is connected with all the nodes present in set $\mathrm{S}$ using star topology network.

3. Central Controller [distributed system] (N) runs a passive scan over all other devices dynamically to achieve information about the status all the devices connected with it and always keep updated information.

4. A node Ni $(\mathrm{i}=1,2,3,4,5, \ldots \ldots \ldots . .$.$) want to store the data to$ any other device $\mathrm{Nj}(\mathrm{j}=1,2,3,4,5, \ldots \ldots$. where $\mathrm{j} \neq \mathrm{i})$ runs an active scan over Central Controller $(\mathrm{N})$ to achieve the information about the status of rest other devices.

5. When the status of all the nodes were obtained, the node that want to store the data will directly choose the node having large storage space and it store the data within that node.

6. Continue step 4 and 5 until data communication over. And the secure communication can be provided with the KF sensor.

7. End

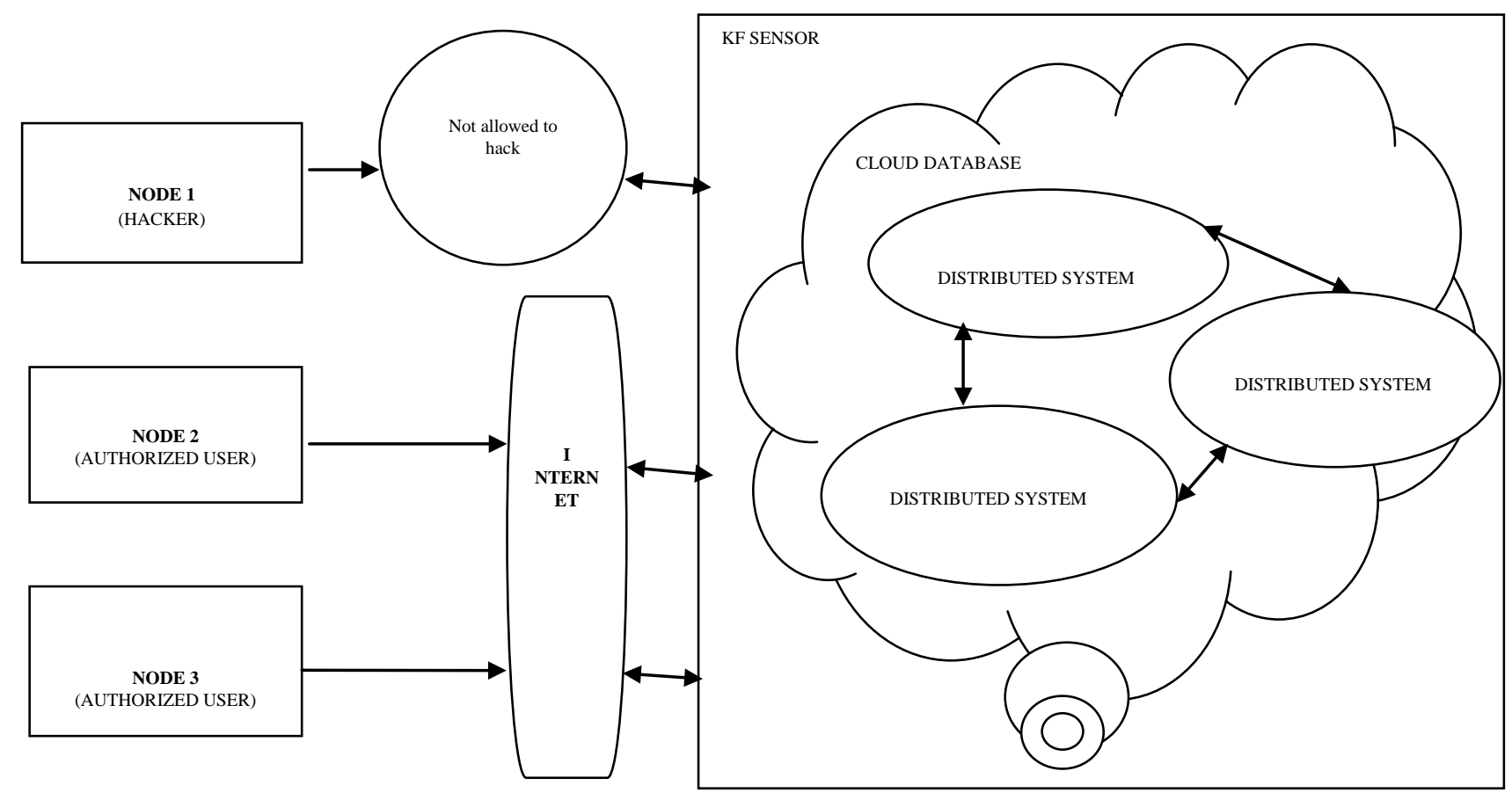

Figure 8: Distributed System Model with Cloud Infrastructure 


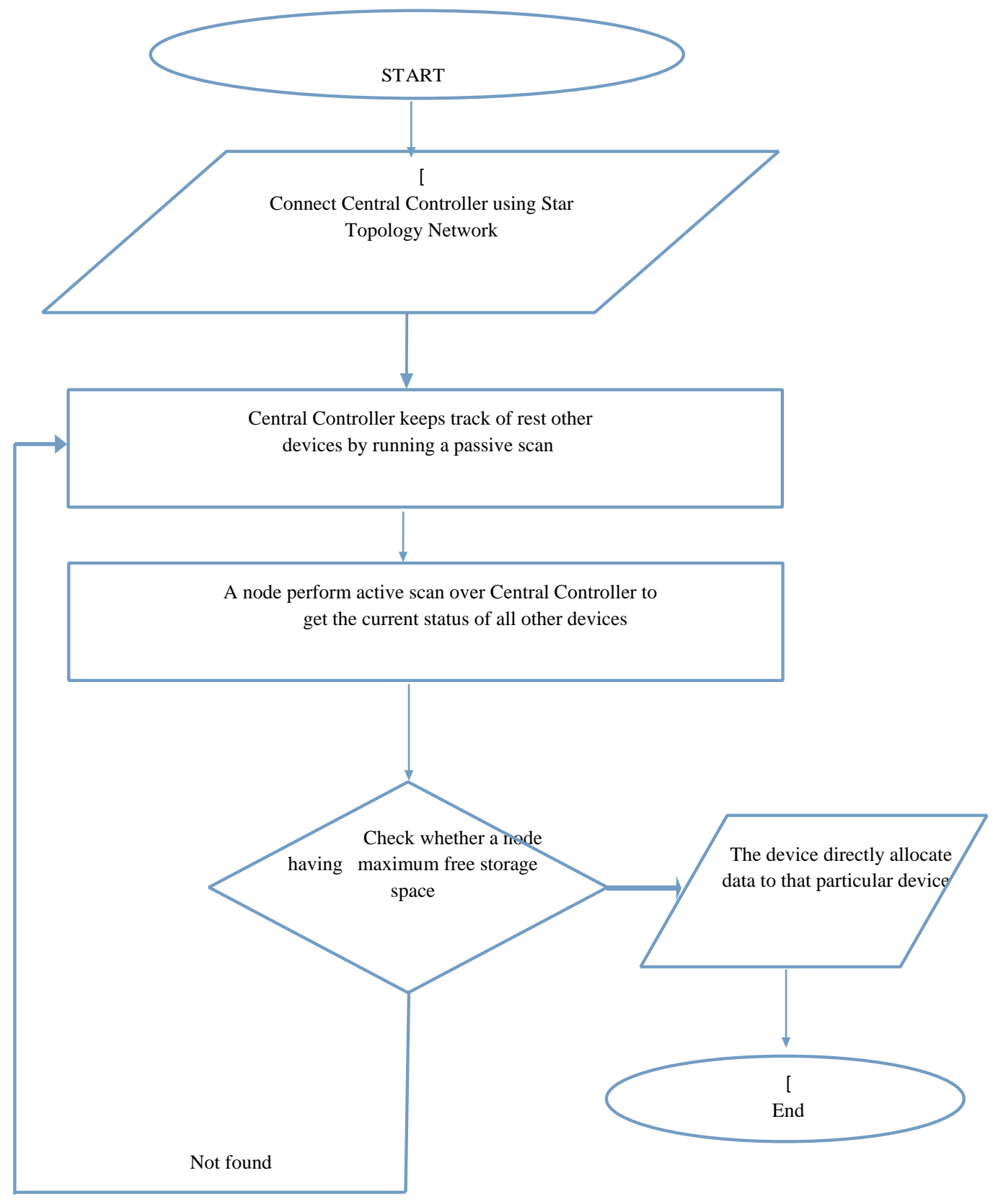

Figure 9: Flowchart of Central Controller Concept

\section{CONCLUSION}

The distributed system as a centralized controller in star topology with the MCC will increase the performance of the mobile device and the battery power of the mobile device. A touchpad device can be used with smart phones via wireless network to overcome the problem of typing in the mobile device. This topology will increase the storage efficiency of the mobile device using the cloud infrastructure. The security of the data stored in the cloud provided with the KF sensor. So all the factor combined together to increase the efficiency of the mobile device and satisfy the user experience in mobile device in higher level.

\section{ACKNOWLEDGEMENT}

I am thankful to Prof. K. Sivakumar and Delsey M J for them guidance and support to pursue this work.

\section{REFERENCES}

[1] P. Theresa Joy and K. Poulose Jacob, "Cooperative Caching Framework for Mobile Cloud Computing”, Global Journals Inc.(USA), Version 1.0, Vol. 13, No. 8, 2013.

[2] S. Shafique Qureshi, "Mobile Cloud Computing as a Future for Mobile Applications-Implementation methods and Challenging Issues”, Proc. of IEEE CCIS, 2011.

[3] P. Bhal, "Advancing the state of mobile cloud computing", Proceedings of the Third ACM workshop on Mobile Cloud Computing and services, Pp. 21-28, 2012.

[4] R. Buyya, C.S. Yeo, S. Venugopal, J. Broberg and I. Brandic, "Cloud Computing and emerging IT platforms: Vision, hype, and reality for 
delivering computing as the 5th utility”, Future Generation Computer Systems, Vol. 25, Pp. 599-616, 2009.

[5] J. Han, W. Susilo and Y. Mu, "Identity-based data storage in cloud computing”, Future Generation Computer Systems, Vol. 29, Pp. 673681, 2013.

[6] A. Kumar, "World of Computing and Security", International journal of Cloud Computing and Services Science, Vol. 1, No. 2, Pp. 53-58, 2012.

[7] P. Zhang Zhao, G. Cao and C. R. Das, "Cooperative caching in wireless P2P networks: design, implementation and evaluation", IEEE Trans Parallel Distributed Syst., Vol. 21, No. 2, Pp. 229-241, 2010. 